\title{
LOCAL DETECTION OF DEFECTS FROM IMAGE SEQUENCES
}

\author{
EWARYST RAFAJŁOWICZ， MAREK WNUK， WoJCIECH RAFAJŁOWICZ \\ Institute of Computer Engineering, Control and Robotics \\ Wrocław University of Technology, Wybrzeże Wyspiańskiego 27, 50-370 Wrocław, Poland \\ e-mail: Ewaryst.Rafajlowicz@pwr.wroc.pl
}

\begin{abstract}
Our aim is to discuss three approaches to the detection of defects in continuous production processes, which are based on local methods of processing image sequences. These approaches are motivated by and applicable to images of hot metals or other surfaces, which are uniform at a macroscopic level, when defects are not present. The first of them is based on the estimation of fractal dimensions of image cross-sections. The second and third approaches are compositions of known techniques, which are selected and tuned to our goal. We discuss their advantages and disadvantages, since they provide different information on defects. The results of their testing on 12 industrial images are also summarized.
\end{abstract}

Keywords: image processing, fractal dimension, morphological operations.

\section{Introduction}

Problems of on-line detection of defects in metals, ceramics and other goods are still challenging, since both earlier successes in research (Davies, 2005; Davies, 2008; Malamas et al., 2003) and the growth of computational power stimulate the need for monitoring more demanding and faster production processes. The main difficulty is in on-line and reliable processing of subsequent images, which partially overlap. Furthermore, defects are frequently small and differ only slightly from their background, while their shapes usually cannot be precisely defined. These features motivate our attempts to elaborate a new method for detecting defects as well as composing and tuning known subalgorithms into efficient methods. In contrast to global methods (see (Rafajłowicz, 2008) and the bibliography cited therein), we put emphasis on local methods, since in certain industrial processes we are faced with global variability of image intensity, which is not a defect indicator, while local variabilities of globally nonuniform intensities are such indicators.

As an example of such a process, which was motivating for the authors, consider a hot metal slab just before entering a hot rolling mill. Images of the slab can be characterized as follows:

1. Defects which are easily visible by the human eye are also visible on images taken by CCD or CMOS cameras, but their contrast is very low (even 10-20 in the scale $[0,255])$.
2. The same images, when observed by an infra-red sensitive camera, reveal a high variability in metal temperature, even for images taken at intervals of 23 seconds. The variability in the temperature translates into the variability in the background on images observed by CCD or CMOS cameras.

3. Furthermore, even the temperature (hence, also the background) of a slab in one image is nonuniform, since its boundaries are colder.

The reader can find discussions on implementation issues of CCD cameras for registering hot metal surfaces in (Dworkin, 2006; O'Leary, 2005).

We discuss three approaches to detecting defects, which take into account the above circumstances. First, a method which is based on the estimation of fractal dimensions of gray levels in cross-sections (see Section 4) is considered. Fractal dimensions were already used in image processing (Conci, 1998), but their use was global in the sense that the box counting dimension was estimated from the entire image. In contrast, we propose to estimate fractal dimensions more locally, for each column (or row) in order to increase sensitivity to small defects. As is known (see, e.g., (Barnsley, 1988)), the fractal dimension of a set, which is composed of subsets having different fractal dimensions, is dominated by the one with the largest fractal dimension. In our case, defects have lower fractal dimensions than their surroundings. Thus, 
the proposed local approach is expected to be much more sensitive to defects than the global one. Additionally, the estimation of fractal dimensions by the correlation method is more accurate and less computationally demanding than the box counting method.

Simultaneously, we discuss two approaches which are compositions of known operations on images, selected and tuned to detecting defects in low contrast industrial images. The first of them (see Section 5) uses the method of finding all local minima and the morphological opening. The second one (see Section 6) consists of adaptive background removing and a special kind of thresholding. The description of each method is accompanied by a simple example which illustrates its performance, while the results of more extensive verifications and comparisons are deferred to the end of the paper.

We emphasize that the methods considered can be applied to the same task of detecting defects, but they are not fully equivalent in the following sense. The method based on estimating fractal dimensions allows only the detection of defects. The method based on morphological operations is able not only to detect defects but also to localize them. The third approach additionally allows the estimation of their areas, moments, etc.

Alternative ways of detecting defects can be based on globally or locally calculated correlations and entropy (Tsai et al., 2003; Skubalska-Rafajłowicz, 2008), but these approaches require further studies before trying to apply them to hot metals.

The paper is organized as follows (see Fig. 1): In the next section we introduce the notations and basic assumptions. Then, an outline of the algorithm for redundancy reduction from overlapping subimages is described. As a

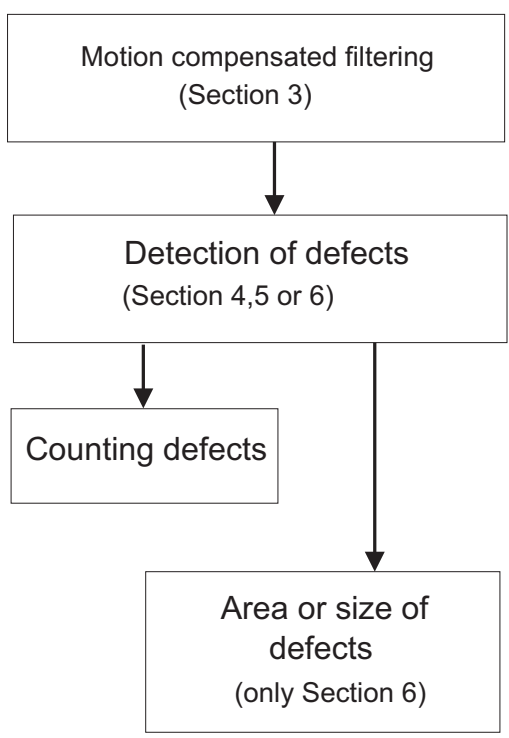

Fig. 1. Flow chart of defect detection steps. result, we obtain initially filtered and nonoverlapping images that can be further processed by algorithms which are described in Sections 4-6. Finally, in Section 7, the results of their empirical verification are presented.

\section{Assumptions}

Images that are considered in this paper are represented by functions $f(x, y)$ of two spatial variables $(x, y)$, where $f(x, y)$ is the intensity of the gray level of the image at a spatial coordinate $(x, y)$. As usual, $x$ and $y$ are coordinates of a pixel and they can take only discrete values, i.e., $x \in\left\{1,2, \ldots, N_{x}\right\}, y \in\left\{1,2, \ldots, N_{y}\right\}$, where $N_{x}$ and $N_{y}$ denote the image width and height, respectively. Time-varying images $f[t](x, y)$ have an added temporal argument $t \in\{1,2, \ldots\}$. Also the range of $f$ is bounded to $[0,1]$ or to integers in the interval $[0,255]$.

The image $g$ provided by a camera is a noisy version of the above, and we assume that

$$
g[t](x, y)=f[t](x, y)+\varepsilon[t](x, y),
$$

where $g[t](x, y)$ is the observed brightness of pixel $(x y)$ at time $t$, while $\varepsilon[t](x, y)$ represents zero-mean noise. A correlation structure of $\varepsilon[t](x, y)$ is postulated only in those sections where it is necessary.

We assume that the observed production line moves in a horizontal direction at a constant speed. Let a positive integer $\Delta>0$ denote the time between the acquisition of two subsequent frames. Without losing generality, we assume that $\Delta$ is the unit of time. During that time the production line moved, and the image of the same portion appears in the next frame $h>0$ pixels further. In other words, for all pixels and time instants we have

$$
f[t](x+h, y)=f[t-1](x, y) .
$$

Note, however, that we admit

$$
g[t](x+h, y) \neq g[t-1](x, y),
$$

since noise is not directly linked to the parts of the monitored production line.

Some remarks concerning images to which our assumptions apply are as follows:

1. Hot metals before entering a rolling mill can be observed by infrared cameras or by industrial cameras, working on the border between visible and infrared light.

2. Monitoring the production of fabrics is a classic example of applying quality monitoring.

3. The results of this paper also apply when discrete products appear in front of a camera. In such a case the reduction of redundant information may not be needed. 


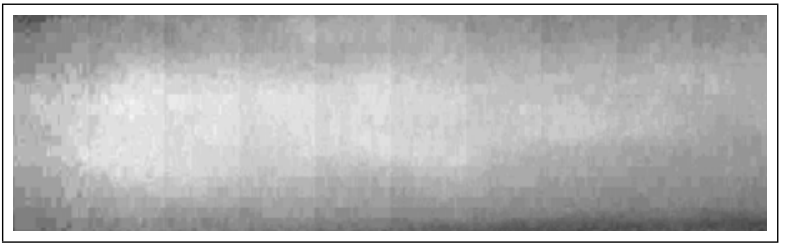

Fig. 2. Example of applying motion compensated filtering to hot metal slabs. Global nonuniformity of the temperature filed is visible.

\section{Motion compensated filtering}

Inequality (3) implies that it is reasonable to filter out noise, taking into account that the corresponding pixels of the clean image $f$ have moved. Clearly, if $h \geq N_{x}$, then motion compensated filtering is not relevant. Thus, to the end of this section we assume that $h<N_{x}$. This means that the same part of the production process is visible at least $m \stackrel{\text { def }}{=}\left\lfloor N_{x} / h\right\rfloor$ times, where $\lfloor a\rfloor$ is the largest integer not exceeding $a$.

From (2) it follows that for unavailable 'clean' images we have

$$
f[t+j](x+j h, y)=f[t](x, y), \quad j=1,2, \ldots, m .
$$

Hence, averaging their noisy counterparts as

$$
m^{-1} \sum_{j=1}^{m} g[t+j](x+j h, y),
$$

we obtain $\hat{f}$ as an estimate of $f$, but with reduced variance. Note that the averaged full image is obtained after every $m$ time steps. Later in this paper subsequent averaged images will be denoted by $\hat{f}[n](x, y), n=1,2, \ldots$ Such images can be further processed in the same way as if the acquisition time were synchronized with the speed of the production line. In other words, each $\hat{f}[n](x, y)$ contains its own portion of the production line. Note that the length of the time interval between $n$ and $n+1$ is equal to $m \Delta$.

Having $\hat{f}[n](x, y)$ at our disposal, we can process it further either column by column, as proposed in the next section, or as a whole image, as described later.

\section{Defect detection by estimating fractal dimensions in cross-sections}

In this section we propose a method of detecting defects in otherwise uniform surfaces by estimating fractal dimensions from images. We refer the reader to (Barnsley, 1988; Falconer, 1990; Ott, 1993; Schuster, 1988) for several definitions of fractal dimensions and for classical methods of their estimation, and to (Chan et al., 1995; Constantine, 1994; Davies, 1999; Kent, 1997; Istas, 1997; Benassi et al., 2002; Skubalska-Rafajłowicz, 2005) for more recent contributions, relevant to this paper.

The following conclusions can be drawn from the analysis of Fig. 3:

1. The brightness levels in cross-sections are very wiggly and one can expect that they can be described by a fractal stochastic process.

2. When a cross-section does not coincide with a defect, then its fractal dimension is relatively high (see the small but frequent twisting and turning movements in the right upper panel of Fig. 3).

3 . In the intervals where cross-sections go through defects, one can observe larger but less wiggly oscillations (see the right lower panel of Fig. 3).

The last conclusion is crucial for the rest of this section, since smaller variations in a curve lead to its smaller fractal dimension. Thus, to estimate fractal dimensions in cross-sections, it suffices to find local minima of the curve, which is composed of the fractal dimensions in crosssections and to set a threshold for the fractal dimension in a local minimum, below which a given cross-section is declared to contain defects.

The key step in successful applications of the above simple idea is in a precise estimation of a selected fractal dimension. For our purposes, we select the correlation
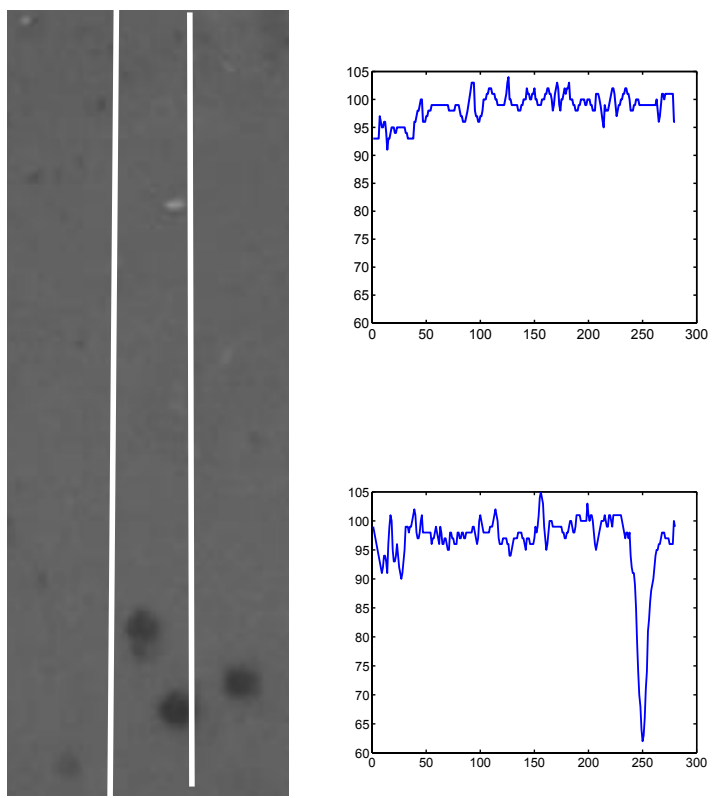

Fig. 3. Left panel - a piece of metal with defects and lines, indicating where cross-sections were selected. Right panelbrightness in the marked cross-sections of the metal surface (the upper right plot corresponds to the left crosssection).

fractal dimension, which is defined below. Then, we shall describe the method of its estimation from samples. 
As mentioned above, we estimate a fractal dimension for each vertical cross-section of images. Thus, subsequent images $\hat{f}[n](x, y), n=1,2 \ldots$ are considered as one long entity. Fix $x$ and denote by $s(y)$ the corresponding column of $\hat{f}[n](x, y)$. We skip the number $n$ of this image for brevity and in order to emphasize that each column in each image is processed in the same way.

We assume $s(y)$ is a stationary stochastic process with a finite variance and write $\gamma(y)=\operatorname{cov}(s(y), s(0))$ for its covariance function at distance $y$. Following (Davies, 1999) we assume a relatively simple model for the covariance function

$$
\gamma(y)=\gamma(0)-c|y|^{2 H}+o(|y|), \quad \text { as } y \rightarrow 0
$$

for certain $c>0$, where $o(|y|)$ denotes terms which decay to zero faster than $|y|$ as $|y| \rightarrow 0$. In the above, $0<H \leq 1$ is the Hurst exponent, which characterizes the smoothness ${ }^{1}$ of trajectories of $s(y)$. For a large class of processes, $H$ is related to the fractal dimension of $s$ as follows:

$$
F_{\text {dim }}(s)=2-H .
$$

Equality (7) holds for a wide subset of second-order stochastic processes. It is not, however, valid for every second-order process. (See the monograph (Adler, 1981), Ch. 8, and (Benassi et al., 2002; Benassi et al., 2003; Tricot, 1995) for sufficient conditions.)

The correlation method (CM) of estimating the Hurst exponent $H$ and by (7) also the correlation fractal dimension can be described as follows (see (Davies, 1999)).

Let $s_{i}$ denote equidistant samples of process $s(i \tau)$, $\tau>0, i=1,2, \ldots, N$. If $\tau=1$, then all pixels in a given cross-section are taken into account. In this case we have $N=N_{y}$. Select the number of lags, $1<M<N$, say, which should be a fraction of $N$. Define the variogram

$$
g_{j}=(N-j)^{-1} \sum_{i=1}^{N-j}\left(s_{i+j}-s_{i}\right)^{2}
$$

$j=1,2, \ldots, M$, which estimates $2(\gamma(0)-\gamma(j \tau))$. According to (6), for $|y|$ small enough,

$$
\log (\gamma(0)-\gamma(y))=2 H \log (|t|)+\text { const. }
$$

Thus, the estimator $\hat{H}$ of the Hurst exponent $H$ is calculated as half of the slope of the linear regression fit of $\log \left(g_{j}\right)$ on $\log (j), j=1,2, \ldots, M$. Finally, the fractal dimension is estimated as $\hat{F}_{\operatorname{dim}}(s)=2-\hat{H}$.

Extensive simulations reported in (Rafajłowicz, 2004) indicate that the estimation errors of the above algorithm can be approximated by the Gaussian distribution with zero mean and dispersion 0.05. As we shall see below, this level of accuracy is in many cases sufficient for detecting defects.

\footnotetext{
${ }^{1}$ For a Gaussian process $H=1$, if $s(\cdot)$ is differentiable.
}

Having estimated fractal dimensions of crosssections, it remains to select a threshold $0<F_{\min }<1$. If the local minimum of the fractal dimensions is below $F_{\text {min }}$, then the existence of defects in the current crosssection is signaled. Equivalently, one can use $H_{\max } \stackrel{\text { def }}{=}$ $2-F_{\min }$ and a signal is given if in the local maximum of $\hat{H}$ we have $\hat{H}>H_{\max }$.

Summarizing, the proposed method runs as follows:

Step 1. Acquire gray levels $s_{i}, i=1,2, \ldots, N$ of pixels of the next cross-section $s$. For $j=1,2, \ldots, M$, calculate $g_{j}$ according to (8).

Step 2. Find the minimum of

$$
\sum_{j=1}^{M}\left(\log \left(g_{j}\right)-a \log (j)\right)^{2}
$$

with respect to $a$ (denote the minimizers by $\hat{a}$ ), which yields

$$
\hat{a}=C_{M}^{-1} \sum_{j=1}^{M} \log \left(g_{j}\right) \log (j),
$$

where $C_{M} \stackrel{\text { def }}{=} \sum_{j=1}^{M} \log ^{2}(j)$ is a constant, which can be precomputed together with $\log (j)$ s.

Step 3. Calculate $\hat{F}_{\operatorname{dim}}(s)=(4-\hat{a}) / 2$ as the estimate of $F_{\operatorname{dim}}(s)$ (or the estimate $\hat{H}(s)=\hat{a} / 2$ of the Hurst exponent).

Step 4. If in the cross-section $s$ the local minimum of $\hat{F}_{\text {dim }}(s)$ s is present and $\hat{F}_{\text {dim }}(s)<F_{\text {min }}$, then declare the existence of defects in the cross-section $s$. (Equivalently, we search for local maxima of $\hat{H}(s)$ and the existence of a defect is declared if $\hat{H}(s)>$ $H_{\min }$.)

Step 5. Repeat from Step 1.

In the above algorithm, $F_{\min }$ (respectively $H_{\min }$ ) is a preselected level, which controls the sensitivity of the method (see Section 7). When tuning the above method, it is more convenient to work with the Hurst exponent curve $\hat{H}(s)$ than with $\hat{F}_{\text {dim }}(s)$, since our eyes locate local maxima more precisely than minima.

Several remarks concerning the above algorithm are as follows:

- As the indicator of the local minimum of $\hat{F}_{\operatorname{dim}}(s)$ at $s$ we take the inequalities $\hat{F}_{\operatorname{dim}}(s-1)>\hat{F}_{\operatorname{dim}}(s)$ and $\hat{F}_{\operatorname{dim}}(s+1)>\hat{F}_{\operatorname{dim}}(s)$.

- The algorithm does not guarantee that $\hat{H} \leq 1$ and $\hat{F}_{\text {dim }} \geq 1$. This can be done by considering the least squares method with constraints. We do not introduce such modifications, since it occurred that a violation of the constraint $\hat{H} \leq 1$ frequently appears exactly when a defect is present (see the example below). 

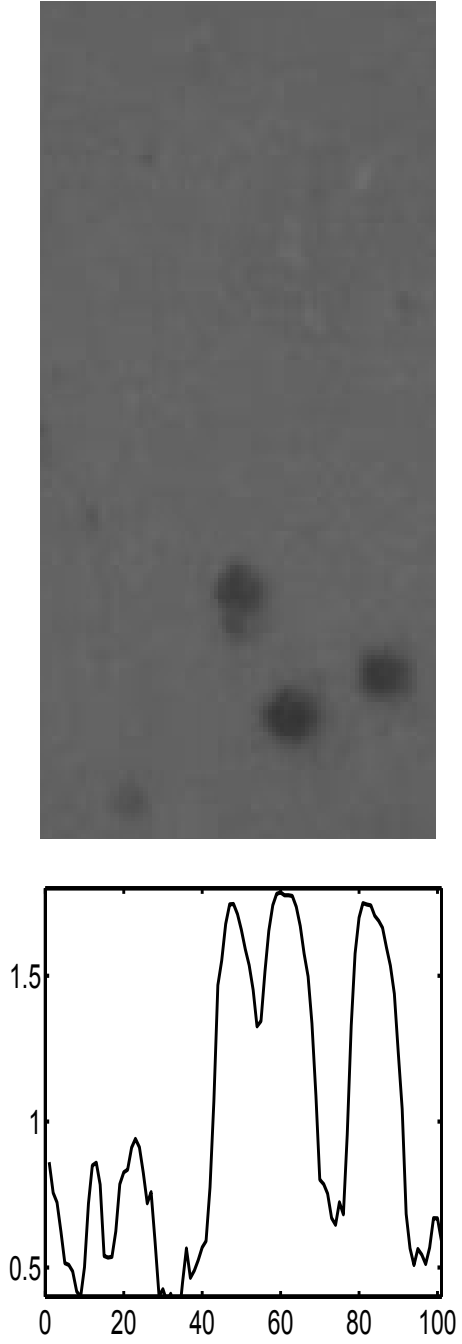

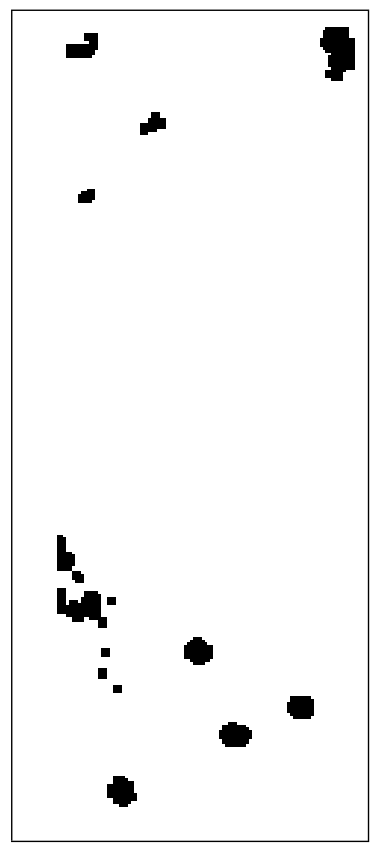

Upper left panel - a hot slab with defects. Bottom left panel - the estimates of the Hurst exponent for each vertical cross-section of the slab (local maxima indicate cross-section with defects).

Upper right panel - defects detected by applying the sequence of morphological operations.

Fig. 4. Illustrative example of the performance of the methods described in Sections 4 and 5.

- The computational complexity of (10) is $O(N)$, since $M$ is a fraction $N$ (usually, $M=N / 10$ ). The calculation time, using a dual core $2.4 \mathrm{GHz}$ computer, for $N=280$ was about $0.0025 \mathrm{~s}$. Assuming a pixel width of $0.4 \mathrm{~mm}$, we can assure on-line monitoring of continuous production processes, which run with at a speed of $20 \mathrm{~cm} / \mathrm{s}$.

The performance of the proposed method is illustrated on an image of a hot metal slab, which is shown in the upper panel of Fig. 4. In the lower panel of this figure the plot of the Hurst exponent estimates in vertical crosssections is shown. Each point of this plot was obtained by estimating the Hurst exponent from one column of pixels of the slab image. According to the proposed algorithm, one should look at local maxima of the Hurst exponents and to select those which are greater than $H_{\min }$ as indicators of defects. The analysis of the curve in the lower panel of Fig. 4 shows that if we take $H_{\min }=0.55$, say, then the peaks precisely point out all the defects which are visible in this slab. It is also clear that this method may overlook a defect if two or more defects are placed exactly one over another. We shall return to the discussion of properties of this method in Section 7.

\section{Morphological technique in defect detection}

As mentioned earlier, industrial images of hot metals frequently have a nonuniform and nonstationary background. For these reasons, the well-known techniques, such as simple thresholding and contouring, fail. Our aim in this section is to propose a combination of morphological operations which detects defects and is robust against the nonuniformity of a background and its nonstationarity.

In the next section we propose an alternative way of overcoming these difficulties, which is based on a special kind of thresholding and adaptive background estimation. 
Our first step is to find all local minima in an image. The rationale behind this step is that local minima are areas in which the temperature is lower than in their surroundings. A lower temperature, in turn, may indicate the presence of a defect. Additionally, nonuniform and nonstationary background does not influence the detection of minima, since this operation is local. It may, however, happen that relatively large areas having the same temperature, which is lower than the surroundings, are erroneously marked as defects. The only drawback of finding all local minima is in a relatively high computational complexity, but the algorithm of van Herk, Gill and Werman (Gill, 1993; Van Herk, 1992) essentially reduces the computational burden.

In order to reduce the number of false positive decisions, one can use a number of tools, but we suggest to stay within the area of morphological algorithms (Davies, 2005; Jahne, 2002; Pratt, 2001) and to use the opening operation.

It should be noted that the opening operation was already applied by Davies (2005, pp. 661-665) in the inspection of cereal grains.

The justification of applying the opening operation also in our case is the following: By selecting a structuring element of an appropriate size, we can control the size of marked areas, which are wiped out. The erosion operator is usually advocated for this purpose, but we prefer the opening operator, combined with local minima, for the following reasons:

- The opening operator completely removes marked areas that are smaller than a prescribed size and approximately restores the areas of remaining objects, since it is a combination of erosion followed by dilation with the same structuring element.

- After the opening operation, the object boundaries become smoother, which is in agreement with shapes of defects in hot metals, since they frequently arise as bubbles caused by gases.

- An algorithm for simultaneous and efficient finding all local minima and performing the opening operation is known (Vincent, 1993).

Specifically, the proposed sequence of morphological operations runs as follows:

Step 1. Find all local minima, which are deeper than a specified level $0 \leq D<255$. Simultaneously, all the surrounding pixels of a given local minimum are also marked if they have the same gray level as the minimum.

Step 2. Apply the opening operation.

Step 3. Count and/or find positions and/or (approximately) evaluate the sizes of defects.
The result of applying Operations 1 and 2 to our test image is shown in Fig. 4 (the upper right panel). As one can notice, local minima indicate points which are identified as defects by a visual inspection. Simultaneously, also points which are not classified as defects are marked, since this is a colder large area (the upper right corner).

\section{Selection of the standard image processing procedures for defect detection}

The basic conditions of defect detection have been formulated on the basis of theoretical and experimental analysis of a real industrial process of continuous casting:

- The images of defects are dark, as a result of worse heat propagation from the inside of the slab.

- The brightness of the slab surface is not uniform. Particularly, the slab boundaries are darker than the slab centre.

- The average brightness of the slab (which reflects its temperature) is time-varying. Nevertheless, assuming a constant brightness on the interval of 1 second seems reasonable.

After some preliminary experiments, the basic prerequisites for selecting image processing procedures have been formulated:

- Gradient-based methods are irrelevant, because the high-frequency noise is comparable to the amplitude of the defects.

- The defects are represented by groups of separate dark spots, rather than connected areas. The elimination of high-frequency components results in decreasing the contrast of the image. Linear low-pass filtering (both in the space and frequency domains) are not applicable. Clustering methods for the aggregation of the subregions of similar local characteristics (texture) are highly recommended.

- The brightness of the slab surface is not uniform, and hence simple thresholding methods cannot be applied for defect detection. Instead, a variable threshold can be used, with the threshold function estimated on-line, based on the short (1 second) sequence of the slab images. The proposed method allows eliminating the main error sources:

- nonuniform brightness of the slab surface (particularly on the slab boundaries);

- time-varying average brightness of the slab surface; 
- permanent artifacts resulting from the image acquisition subsystem (e.g., stains on the lens and optical filters).

The suggested stages of the image processing system for the estimation of defects in the metal slab, resulting from the former analysis, are as follows:

- early processing,

- segmentation (thresholding),

- secondary processing (aggregation),

- parametrization of the extracted blobs (the area and shape of a blob, an estimated volume of the underlying defect).

A more detailed description of the enumerated stages follows.

6.1. Early processing. The first problem is to define the ROI (Region of Interest) - a rectangular area of the frame, which corresponds to the visible slab surface. During system calibration this can be performed on the basis of the Hue analysis of the color image (which is a very distinctive feature of the slab). Better results can be obtained by applying an adaptive ROI extraction at every one second interval or even on every frame basis.

All further processing is limited to the defined ROI, which significantly reduces the data volume. Applying a color (RGB) camera allows us to select the signal which is most relevant to defect extraction. Experiments have shown that the Red plane is the best, while the Bluethe worst. In the described experiment we assumed using only the Red component of the RGB image. Note that it is possible to replace the color camera with a grey-scale one, equipped with a Red optical filter, which allows us to reduce both the equipment cost and the amount of transmitted and acquired data.

The requirement of on-line processing yields choosing thresholding as the segmentation method. The aforementioned nonuniformity of the image brightness, as well as its time-dependent nature, requires an adaptive version of the variable-threshold method. It is well known that the variable thresholding segmentation is equivalent to the composition of background subtraction and uniform thresholding.

The slab image is created by the emitted light, rather than the reflected one. In this case we have to consider an additive model of image formation. Assuming that the average value of the nonuniformity is close to zero in a certain time interval $\left(t_{1}, t_{2}\right)$, we can use the slab image, averaged in that interval, as the correction image $f_{c}(x, y)$. Hence we can perform a subtractive shading correction:

$$
g(x, y)=f(x, y)-f_{c}(x, y) .
$$

Choosing an appropriate constant threshold $t$, we can perform a simple segmentation of the corrected image:

$$
g_{t}(x, y)=\left\{\begin{array}{c}
1 \quad \text { if } g(x, y) \geq t, \\
0 \quad \text { if } g(x, y)<t,
\end{array}\right.
$$

which is equivalent to variable thresholding of the original image:

$$
g_{t}(x, y)= \begin{cases}1 \quad \text { if } f(x, y)<t(x, y), \\ 0 \quad \text { if } f(x, y) \geq t(x, y),\end{cases}
$$

with the threshold function

$$
t(x, y)=t+f_{c}(x, y) .
$$

We should implement a moving average of the frames in order to follow slow changes in the average slab brightness. The brute-force method requires a large amount of memory space and processing power. We implemented an iterative algorithm of the exponentially weighted moving average, which requires only one frame buffer for the correction image. At the moment $n \tau_{c}$ (where $\tau_{c}$ denotes the image acquisition period) we get a consecutive image $f[n](x, y)$. The new correction image $f_{c}[n](x, y)$ is formed as a convex combination of the former correction image and the current frame:

$$
f_{c}[n](x, y)=(1-\varepsilon) f_{c}[n-1](x, y)+\varepsilon f[n](x, y),
$$

where $0<\varepsilon<1$ determines the degree of smoothing (for $\varepsilon$ closer to zero the filtering is more intensive). In our context it is expedient to provide also the following interpretation of $\varepsilon$ by an analogy to the first-order lowpass $\mathrm{RC}$ filter, for which the time constant is equal to $R C$, where $R$ is the resistance and $C$ is the capacitance of the filter elements: The $\varepsilon$ parameter defines the time constant of the resulting first-order low-pass filter as

$$
T=-\frac{\tau_{c}}{\ln (1-\varepsilon)},
$$

where $\tau_{c}$ is the frame acquisition period. Note that (16) suggests that in selecting $\varepsilon$, one should take into account the frame acquisition rate. In our examples, $\varepsilon=1 / 8$ was selected for $\tau_{c}=0.16 \mathrm{~s}$, which resulted in the time constant $T=1.2 \mathrm{~s}$. Thus, after about $5 T=6 \mathrm{~s}$, the filter forgets old frames.

6.2. Segmentation. As shown before, after shading correction, segmentation can be performed with a fast and simple method of thresholding. The method is based on a-priori known distributions of both the object and the background brightness, and the expected total objects size (e.g., Bayes classifiers) cannot be used for the lack of $a$ priori data. 


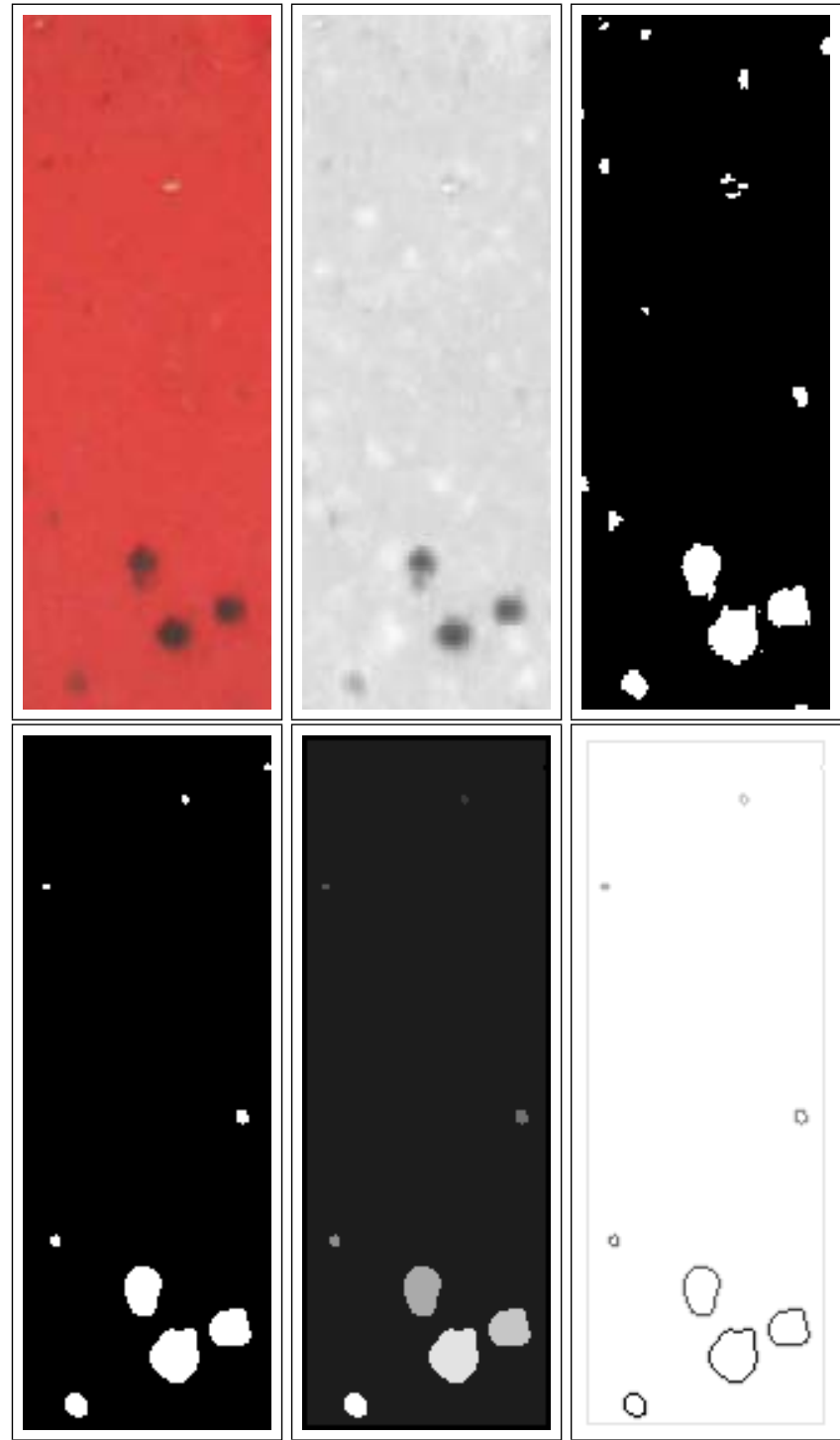

Fig. 5. Exemplary results. Upper panels - original, shading correction, segmentation. Bottom panels - aggregation, blob coloring, contours.

The only information we can rely on is that the defects are represented by connected areas with brightness lower than the surrounding background. The proposed method of double thresholding (Rosenfeld, 1982) is local (unlike simple thresholding, which is point-based). It consists in defining two thresholds:

- radical one: $t_{r}$ (the pixels darker than $t_{r}$ belong to the defect area unconditionally),

- liberal one: $t_{l}$ (the pixel below $t_{l}$ are accepted if in their neighbourhood there exist pixels of the former kind).

In our case (dark objects on a brighter background), $t_{r}<t_{l}$. Let us denote by $b(x, y)$ a binary image at pixel $(x, y)$, which is the output of the following double thresholding procedure:

1. Denote by $S_{x y}$ a neighbourhood of pixel $(x, y)$.

2. Set $b(x, y)=1$ if $g(x, y)>t_{l}$, and simultaneously one can find a pixel $(\xi, \psi)$ in $S_{x y}$ such that $g(\xi, \psi)>$ $t_{r}$.

3. Set $b(x, y)=1$ if for every $(\xi, \psi)$ from $S_{x y}$ we have $g(\xi, \psi)<t_{r}$.

The described method is only slightly more complex than plain thresholding, but is very robust and does not require thorough tuning of the $t_{r}$ and $t_{l}$ thresholds. It should be noted that this approach, called hystheresis thresholding, was used in (Davies, 2005; pp. 209-212), for thinning detected edges. 
6.3. Secondary processing. The result of thresholding is a binary image representing the slab defects as well as the imperfections of the image acquisition system. A practised observer can easily distinguish between them. In fact, a kind of aggregation algorithm based on relative nearness of the subareas can solve the problem.

The median filtering allows us to assure a proper aggregation. In general, this method is computationally complex, but in the case of a binary image it can be reduced to simple voting (a majority filter). It can be implemented as a uniform linear filter with simple thresholding on the level of half the area of the filter neighbourhood. Linear (FIR) filters are efficiently implemented in DSPs and other machines supporting the MAC (Multiply and Accumulate) instruction.

6.4. Parametrization. The silhouettes, extracted by segmentation, must be described by some parameters (area, eccentricity, location within the image frame, etc.). These parameters can be calculated by means of geometrical moments. Software implementation of this method can be very efficient (a single image scan is sufficient). Moreover, even hardware solutions are possible (using the pipelined architecture, see (Wnuk, 2008)).

The method of moments (Gonzalez, 1977) provides not only the location of the object represented by the silhouette but also many parameters describing its shape (e.g., Hu moments) (Hu, 1962). The parameters based on normalized central moments are translation-, rotation-, and scale-invariant and thus can be used for object classification.

6.5. Implementation example. Tests of the proposed image processing sequence were performed with many real, industrial image sequences of the moving metal slab. The Khoros environment (2.2.0.0 version) was used in the experimental implementation. The original color image is redundant in the described case. For further processing, only the Red component was used. After shading correction the defects are clearly visible.

Double thresholding $\left(t_{r}=-20\right.$ and $\left.t_{l}=-8\right)$, with the neighbourhood radius of 3 , results in a binary image with groups of spots showing the defects. The results of early processing and segmentation are presented in Fig. 5 (upper panels).

Aggregation with the majority filter (a linear implementation of the median for binary images), with the neighbourhood radius of 3 , results in clear silhouettes of the defects.

The four-connected blob coloring algorithm was used for silhouette indexing. The results of secondary processing and object indexing are presented in Fig. 5 (lower panels).
Finally, the blobs parameters, calculated using the method of moments, are shown in the exemplary printout (Fig. 6).

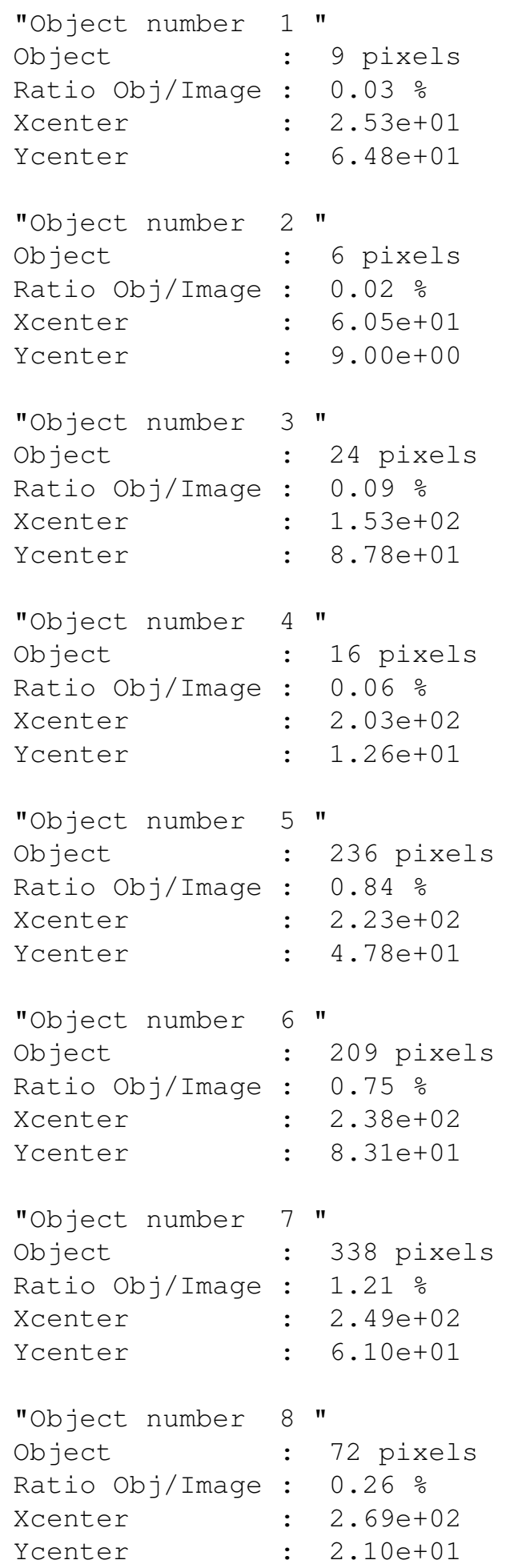


Table 1. Comparison of tasks that can be realized by the discussed methods. The column 'Size' refers to possibilities of measuring a diameter, area, etc. 'yes/no' means that the task can be realized with a limited accuracy only.

\begin{tabular}{|c|c|c|c|}
\hline \multirow{2}{*}{ Method } & \multicolumn{3}{|c|}{ Defects } \\
\cline { 2 - 4 } & Counting & Locating & Size \\
\hline \hline FracDim & yes & no & no \\
\hline Morph. & yes & yes & yes/no \\
\hline DT & yes & yes & yes \\
\hline
\end{tabular}

\section{Discussion and comparisons}

Before entering into details of comparisons, we discuss properties and sources of errors of each method.

Firstly, we concentrate on false decisions.

1. The method based on the estimation of the correlation fractal dimension may overlook a defect if it is hidden behind another one, i.e., when their $x$-coordinates are almost the same while the $y$ coordinates are different. This drawback can be reduced to some extent by repeating the same procedure to all rows of the image and taking a larger number of defects detected in rows and columns. This approach, however, increases the computational burden. In fact, the situation here is similar to the one which is observed when the method of a lateral histogram is used (Davies, 2005). False detections are possible when additional local maxima of the fractal dimension curve arise as a result of the estimation errors.

2. The sequence of morphological operations may overlook defects which are smaller than the size of the structuring element used for erasing noise, speckles, etc. It seems that this kind of errors is unavoidable for this method, since by reducing the size of the structuring element we simultaneously increase the number of falsely detected defects.

3. The method of double thresholding may fail to detect small defects with low contrast between the defect and its surrounding.

Clearly, all three methods can detect nonexistent defects, when drops of cooling liquid or other objects are present, which have visual characteristics similar to those of defects.

As mentioned earlier, the three methods are not equivalent (see Tab. 1) for the following reasons:

(a) Fractal dimensions along columns allow counting defects but cannot locate them without an additional computational burden. (b) Local minima followed by opening allow counting and locating defects, but erosion followed by dilation, although restores a general shape, introduces unpredictable changes in the size of a defect. Thus, this method can be used for measuring sizes and areas of defects only approximately.

(c) Double thresholding allows counting, locating and measuring defects.

Summarizing, all three methods provide the number of defects and at this level they are compared below. For comparisons, we used 12 slabs (five directly before the one shown in Fig. 4 and six after it). As the ground truth we used the number of defects counted by our eyes (see the last column in Tab. 2). The numbers of defects found by each of the discussed methods are summarized in Tab. 2, where FracDim stands for the method described in Section 4, Norph. is the abbreviation for the method discussed in Section 5 and $D T$ for the one from Section 6.

We do not have enough empirical data for calculating the receiver operating characteristic of each method. However, from Tab. 2 we can estimate the lower bounds for the probability of a false positive (FP) decision and a false negative (FN) decision, which are presented in Tab. 3 as a summary of all 12 slabs. We can also investigate the

Table 2. Defects detected by the three methods in the sequence of 12 slabs.

\begin{tabular}{|c|c|c|c|c|}
\hline $\mathrm{Nr}$ & FracDim & Morph. & DT & True \\
\hline \hline 1 & 20 & 8 & 8 & 21 \\
2 & 19 & 22 & 16 & 21 \\
3 & 20 & 40 & 24 & 21 \\
4 & 14 & 24 & 24 & 17 \\
5 & 16 & 14 & 13 & 16 \\
6 & 14 & 11 & 8 & 16 \\
7 & 15 & 12 & 13 & 16 \\
8 & 17 & 12 & 15 & 17 \\
9 & 18 & 17 & 25 & 21 \\
10 & 15 & 8 & 17 & 15 \\
11 & 20 & 30 & 7 & 18 \\
12 & 18 & 3 & 3 & 17 \\
\hline Sum & 206 & 201 & 173 & 216 \\
\hline
\end{tabular}

variability of the discussed methods to changes of their parameters. The methodology of our studies was the following for each method:

(a) a crucial parameter, influencing the number of detected defects in a dominating way, was selected,

(b) numbers of detected defects from images of 12 slabs were summed. 
The results of these experiments are summarized in Tab. 4, where the $H_{\text {min }}$ parameter was selected for the FracDim method, the minimal depth of local minima was used in the Morph. method and a radical threshold $t_{r}$ in the DT method.

Additionally, the sensitivity of the defects' areas detected by the DT method to changes of $t_{r}$ were investigated. In this case the sensitivity coefficient, $\kappa$, say, is measured as StdDev/Avg, where Avg is the average of the defects' areas detected for a certain range of $t_{r}$ changes, while StdDev is the standard deviation of these areas. A summary of the results for the defects' areas collected from all 12 images is the following: For $t_{r}$ ranging from -19 to -22 , the sensitivity $\kappa$ was $5.27 \%$, while for $t_{r}$ in the range from -15 to -19 , the sensitivity was $4.42 \%$. Thus, the DT method keeps estimated areas of detected defects at a stable level. For aforementioned reasons we cannot repeat these investigations for the FracDim and Morph. methods.

The following conclusions are suggested by the above empirical studies:

1. The FracDim method provides the numbers of detected defects, which are close to the true ones. Simultaneously, it is relatively easily tuned, since the variability to changes of $H_{\min }$ is relatively low.

2. The Morph. method behaves well in most cases but it may happen that the number of false positive detections is almost equal to the number of true defects (see row 3 in Tab. 2). A fine tuning of the Depth parameter is required, otherwise one can expect large FN or FP errors.

3. The DT method has a tendency to underestimate the number of detected defects, but omitted defects have small areas and for this reason it is stable in estimating the total area of defects. Its tuning is also relatively easy (small variability with respect to changes in $t_{r}$ ). One can wonder what the reason of false positive detections of this method is. A deeper analysis of Cases 3, 4, 9, 10 in Tab. 2 reveals that the DT method can split larger defects into parts, leading to an increase in the number of detected defects, but this does not significantly distort the estimated areas.

\section{Conclusions}

A method of motion compensated filtering was presented. As a result of its application, we obtain subsequent images, which are filtered and do not contain redundant information. These images are then processed in order to detect defects. Three methods, which are dedicated for detecting low contrast defects, were tuned for and tested on industrial images of hot metal surfaces. All of them
Table 3. Estimated lower bounds for the probabilities of FP and FN decisions, estimated from 12 slabs.

\begin{tabular}{|c|c|c|}
\hline Method & FP & FN \\
\hline \hline FracDim & $\geq 0.06$ & $\geq 0.015$ \\
\hline Morph. & $\geq 0.25$ & $\geq 0.18$ \\
\hline DT & $\geq 0.09$ & $\geq 0.28$ \\
\hline
\end{tabular}

Table 4. Variability of the number of defects to changes in parameters (see explanations in the text).

\begin{tabular}{c|c|c|c|c|c|c|}
\cline { 2 - 7 } FracDim & $H_{\text {min }}$ & 0.4 & 0.45 & 0.5 & 0.55 & 0.6 \\
\cline { 2 - 7 } & Total & 207 & 206 & 206 & 201 & 196 \\
\cline { 2 - 7 } Morph. & Depth & 8.0 & 10.0 & 11. & 12. & 14. \\
\cline { 2 - 7 } & Total & 270 & 234 & 201 & 177 & 150 \\
\cline { 2 - 7 } DT & $t_{r}$ & -18 & -19 & -20 & -21 & -22 \\
\cline { 2 - 7 } & Total & 180 & 176 & 173 & 172 & 166 \\
\cline { 2 - 7 } & & & & & &
\end{tabular}

proved their abilities of detecting most of the larger defects. On the other hand, each method may have left some defects undetected. Additionally, the method based on double thresholding proved their stability in estimating areas of defects. In a more demanding application, it seems reasonable to apply selected two or even all three methods simultaneously.

\section{Acknowledgements}

The authors would like to express their sincere thanks to the anonymous referees for their helpful comments and suggestions. This work was supported by a research and development grant of the Polish Ministry of Science and Higher Education between 2007 and 2009.

\section{References}

Adler J.R. (1981). The Geometry of Random Fields, Wiley, Chichester.

Barnsley M. (1988). Fractals Everywhere, Academic Press, New York, NY.

Benassi A., Cohen S., Istas J. (2002). Identification and properties of real harmonizable fractional levy motions, Bernoulli 8(1): 97-115.

Benassi A., Cohen S., Istas J. (2003). Local self-similarity and Hausdorff dimension, Comptes Rendus Mathematique 336(3): 267-272.

Chan G., Hall P. and Poskitt D. S. (1995). Periodogram-based estimators of fractal properties, Annals of Statistics 23 (5): 1684-1711.

Conci A., Proenca C.B. (1998). A fractal image analysis system for fabric inspection based on a box-counting method. Computer Networks and ISDN Systems 30(2021): 1887-1895. 
Constantine A.G. and Hall P. (1994). Characterizing surface smoothness via estimation of effective fractal dimension, Journal of the Royal Statistical Society: Series B $\mathbf{5 6}$ (1): 97-113.

Davies E. R. (2005). Machine Vision: Theory, Algorithms, Practicalities, 3rd Edn., Academic Press, San Francisco, CA.

Davies E. R. (2008). A generalised approach to the use of sampling for rapid object location, International Journal of Applied Mathematics of Computer Science 18(1): 7-19.

Davies S. and Hall P. (1999). Fractal analysis of surface roughness by using spatial data, Journal of the Royal Statistical Society: Series B 61 (1): 3-37.

Dworkin S.B. and Nye T.J. (2006). Image processing for machine vision measurement of hot formed parts, Journal of Materials Processing Technology 174 (1-3): 1-6.

Falconer K. (1990). Fractal Geometry, Wiley, New York, NY.

Gonzalez R.C. and Wintz P. (1977). Digital Image Processing, Addison-Wesley, Reading, MA.

Hu M.K. (1962) Visual pattern recognition by moment invariants, IEEE Transactions on Information Theory 6(2): 179187.

Kent J.T. and Wood A.T. (1997), Estimating the fractal dimension of a locally self-similar Gaussian process by using increments, Journal of the Royal Statistical Society: Series B 59 (3): 679-699.

Istas J. and Lang G. (1997). Quadratic variations and estimation of the local Hölder index of a Gaussian process, Annales de I'Institut Henri Poincare (B) Probability and Statistics 33 (4): 407-436.

Jähne, B. (2002). Digital Image Processing, Springer-Verlag, Berlin/Heidelberg.

Gill J. Y. and Werman M. (1993). Computing 2-D min, median and max filters, IEEE Transactions on Pattern Analysis and Machine Intelligence 15(5): 504-507.

O’Leary P. (2005). Machine vision for feedback control in a steel rolling mill, Computers in Industry 56(8-9): 997-1004.

Malamas E.N., Petrakis E. G. M., Zervakis M., Petit L. and Legat J-D. (2003). A survey on industrial vision systems, applications and tools, Image and Vision Computing 21(2): 171-188.
Ott E. (1993). Chaos in Dynamical Systems, Cambridge University Press, Cambridge.

Pratt P.K. (2001). Digital Image Processing, 3rd Edn., Wiley, New York, NY.

Rafajłowicz E. (2008). Testing homogeneity of coefficients in distributed systems with application to quality monitoring, IEEE Transactions on Control Systems Technology 16(2): 314-321.

Rafajłowicz E. (2004) Testing (non-)existence of input-output relationships by estimating fractal dimensions, IEEE Transactions Signal Processing 52(11): 3151-3159.

Rosenfeld A. and Kak A.C. (1982). Digital Picture Processing, Academic Press, Inc., Orlando, FL.

Schuster H.G. (1988). Deterministic Chaos, VGH Verlagsgesellschaft, Weinheim.

Skubalska-Rafajłowicz E. (2005). A new method of estimation of the box-counting dimension of multivariate objects using space-filling curves, Nonlinear Analysis 63 (57): 1281-1287.

Skubalska-Rafajłowicz E. (2008). Local correlation and entropy maps as tools for detecting defects in industrial images, International Journal of Applied Mathematics and Computer Science 18(1): 41-47.

Tricot C. (1995). Curves and Fractal Dimension, Springer, New York, NY.

Tsai D.-M., Lin C.-T., Chen J.-F. (2003). The evaluation of normalized cross correlations for defect detection, Pattern Recognition Letters 24 (15): 2525-2535.

Wnuk M. (2008). Remarks on hardware implementation of image processing algorithms, International Journal of Applied Mathematics and Computer Science 18(1): 105-110.

Van Herk M. (1992). A fast algorithm for local minimum and maximum filters on rectangular and octagonal kernels, Pattern Recognition Letters 13(7): 517-521.

Vincent L. (1993). Grayscale area openings and closings, their efficient implementation and applications, Proceedings of the EURASIP Workshop on Mathematical Morphology and its Applications to Signal Processing, Barcelona, Spain, pp. 22-27.

Received: 9 December 2007

Revised: 19 April 2008 